OLECHOWSKI, Thomas

DOI: 10.15170/DiKE.2020.04.01.15

Universitätsprofessor, Universität Wien

Obmann der Kommission für Rechtsgeschichte Österreichs

der Österreichischen Akademie der Wissenschaften

\title{
Die Anfänge der europäischen Verfassungsgerichtsbarkeit ${ }^{1}$
}

\section{The Beginnings of the European Constitutional Jurisdiction}

In the field of constitutional jurisdiction, there are two models: the American model, which dates back to the late 18th century, in the early years of the United States. And the European model that is often associated with Hans Kelsen, the "architect" of the Austrian constitution of 1920. However, the Austrian Constitutional Court is not the first of its kind, the Constitutional Court of Czechoslovakia is a few months older, but it hardly showed any activity. The triumphal march of this model began after 1945 and is a part of most European constitutions today. The article examines the beginnings of this European model and explains its basic principles.

Keywords: constitutional jurisdiction, judicial review, Austrian Constitutional Court, Hans Kelsen

\section{Kleine Ursache - große Wirkung}

Als Thomas Jefferson am 4. März 1801 sein Amt als dritter Präsident der Vereinigten Staaten antrat, musste er feststellen, dass sein Amtsvorgänger, John Adams, noch buchstäblich bis zur letzten Sekunde Ernennungen von Bundesrichtern vorgenommen hatte, dass Adams aber nicht mehr dazu gekommen war, alle Ernennungsurkunden auch den betreffenden Personen zuzustellen. Jefferson wies daher seinen neuen Secretary of State, James Madison, an, diese bereits unterzeichneten Ernennungsdekrete nicht zuzustellen, wogegen einer der nun-also-doch-nicht-ernannten Richter, William Marbury, Klage beim Supreme Court der Vereinigten Staaten erhob. Dieser wies jedoch die Klage am 24. Februar 1803 wegen Unzuständigkeit zurück. Wohl sehe der Judiciary Act von 1789 in Artikel 13 vor, dass der Supreme Court in derartigen Fällen das zuständige Gericht sei, doch stünde diese Gesetzesbestimmung im Widerspruch zur Verfassung, die die Kompetenzen des Supreme Court abschließend regle, und dürfe daher nicht angewendet werden. ${ }^{2}$

Der hier skizzierte Sachverhalt im Fall „Marbury vs. Madison" ist heute weitgehend in Vergessenheit geraten. Berühmtheit hat jedoch die Begründung erlangt, mit der der Supreme Court

\footnotetext{
${ }^{1}$ Der Beitrag ist die redigierte Version des online Vortrags vom 17. April 2020 in Pécs an der Wissenschaftlichen Sitzung der Dezső-Márkus-Forschungsgruppe für Vergleichende Rechtsgeschichte. Eine englische Version dieses Beitrages erschien in: MADSEn, Mikael Rask - THORnhILl, Chris (Hrsg): Law and the Formation of Modern Europe: Perspectives from the Historical Sociology of Law. Cambridge 2014, 77-95.

2 STOURZH, Wege zur Grundrechtsdemokratie 66 f; PAUlsOn, Constitutional Review 226; HAASE - STRUGER, Verfassungsgerichtsbarkeit in Europa 22 f; HELLER, Der Verfassungsgerichtshof 37.
} 
zu seinem Urteil gelangte, weil er sich hier erstmals zur Frage äußerte, wie mit Gesetzen zu verfahren sei, die im Widerspruch zur Verfassung stünden. Und er kam zu dem Schluss: „Die $V$ erfassung ist entweder ein böchstes Gesetz. (superior, paramount law), das auf gewöhnliche Art und Weise nicht abgeändert werden kann, oder sie steht auf derselben Stufe wie gewöhnliche Gesetze und kann so wie andere Gesetze geändert werden, sobald die Legislative dies wünscht. Wenn die erste Alternative wabr ist, dann ist ein Gesetzgebungsakt, der der Verfassung widerspricht, kein Gesetr. Wenn die zweite Alternative wahr ist, dann sind geschriebene Verfassungen absurde Versuche des Volkes, eine Gewalt zu beschränken, die von ibrem Wesen her nicht beschränkbar ist. Sicherlich haben jene, die Verfassungen entworfen haben, sie als fundamentale und böchste Normen der Nation konzipiert, und konsequenterweise muss es die Theorie in einem solchen Staat sein, dass ein Gesetzgebungsakt, der der Verfassung widerspricht, nichtig (void) ist.”’

Mit dieser Feststellung der Nichtigkeit von verfassungwidrigen Gesetzen im Fall „,Marbury vs. Madison" wird allgemein der Ursprung des amerikanischen Modells der Verfassungsgerichtsbarkeit angesetzt. Natürlich, so ist hinzuzufügen, hatte auch dieser Ursprung eine lange Vorgeschichte, die bis ins England des 17. Jahrhunderts zurückreicht. Und schon 1766 hatte ein County Court der britischen Kolonie Virginia entschieden, dass ein Gesetz (in diesem Fall: der berühmte Stamp Act 1865) keine Geltung beanspruchen dürfe, wenn es verfassungswidrig sei. ${ }^{4}$ Die Bedeutung der Entscheidung im Fall „Marbury vs. Madison “liegt aber darin, dass das Prinzip der judicial review erstmals auch vom höchsten Gericht der Vereinigten Staaten anerkannt wurde und seine Prinzipien bis heute praktisch unverändert geblieben sind.

Mit dieser judicial review kommt den amerikanischen Gerichten eine politisch sehr bedeutende Rolle zu, die die Diskussion in Europa schon sehr früh beeinflusste. Durchsetzen konnte es sich nur in wenigen Ländern; eines der frühesten Beispiele ist der oberste Gerichtshof von Norwegen, der Højesterett, der bereits 1814 ein entsprechendes Urteil fällte. In Dänemark war das Prinzip der judicial review seit 1912 anerkannt, doch erst im Jahr 1999 wurde ein vom dänischen Parlament erlassenes Gesetz vom dänischen Højesteret als verfassungswidrig bezeichnet. ${ }^{5}$ Von den vielen Gegnern einer judicial review sei hier nur Otto von Bismarck genannt, der 1863, zur Zeit des preußischen Verfassungskonfliktes, erklärte, dass "die politische Zukunft des Landes" nicht „von dem einzelnen Urteilsspruche eines Gerichts, wie er sich nach der subjektiven Ansicht der Stimmenden herausstellt", abhängen dürfe. ${ }^{6}$ Aber letztlich konnte sich das Prinzip der judicial review auch in Deutschland durchsetzen; 1921 erst zögerlich, 1925 dann in aller Bestimmtheit erklärte das deutsche Reichsgericht, das oberste Gericht in Zivil- und Strafsachen, dass die Vorschriften der Reichsverfassung den Richter dazu „nöthigen, (...) die widersprechenden Bestimmungen des späteren Gesetzes außer Anwendung zu lassen. "Praktische Konsequenzen hatte dies jedoch nicht: Weder in dem genannten Fall noch sonst in einem Fall bis 1933 wurde jemals ein Reichsgesetz vom Reichsgericht für verfassungswidrig erklärt. ${ }^{8}$

\footnotetext{
${ }^{3}$ Us-Supreme Court, Marbury vs. Madison, Entscheidung vom 2. Februar 1803, zitiert nach UROFSKY, Documents of American Constitutional and Legal History No 46; vgl auch PAULSON, Constitutional Review 228.

${ }^{4}$ STOURZH, Wege zur Grundrechtsdemokratie 37; PAUlSON, Constitutional Review 225.

${ }^{5}$ HAASE - STRUGER, Verfassungsgerichtsbarkeit in Europa 229, 232.

${ }^{6}$ Bismarck am 22.4.1863 im preußischen Abgeordnetenhaus, zitiert nach TRIEPEL, Bericht 9.

${ }^{7}$ Urteil vom 4.11.1925, RGZ 111, 320-335, hier 323.

8 Siehe für Details STOLlEIS, Judicial Review; SELlerT, Richterliches Prüfungsrecht 1053; HAASE - STRUGER, Verfassungsgerichtsbarkeit in Europa 78.
} 


\section{Der österreichische Verfassungsgerichtshof und Hans Kelsen}

Dem amerikanischen Modell der Verfassungsgerichtsbarkeit wird in der Staatsrechtslehre ein zweites Modell gegenübergestellt, das deutlich jüngeren Ursprungs ist und das manchmal als europäisches Modell, manchmal als österreichisches Modell, manchmal als Kelsen-Modell bezeichnet wird. ${ }^{9}$ Damit ist ein eindeutiger Hinweis gegeben auf die von Hans Kelsen maßgeblich mitgestaltete, österreichische Bundesverfassung aus dem Jahr 1920, die eine gänzlich andere Verfassungsgerichtsbarkeit vorsah, bei der nämlich diese Kompetenz bei einem einzigen Verfassungsgerichtshof konzentriert war.

Allerdings, so wird eingewendet, war das österreichische Bundes-Verfassungsgesetz vom 1. Oktober 1920 nicht die älteste Verfassungsurkunde mit einem derartigen verfassungsgerichtlichen Modell. Sieben Monate älter war die Verfassungsurkunde der Tschechoslowakei vom 29. Februar 1920, die ebenfalls einen einzigen Verfassungsgerichtshof vorsah und den übrigen Gerichten eine judicial review verbot. ${ }^{10}$ Nun ist bekannt, dass die von Kelsen begründete Schule, die Reine Rechtslehre, auch in der Tschechoslowakei, namentlich in Brno, dem Sitz des tschechoslowakischen Verfassungsgerichts, viele Anhänger hatte. Doch waren diese, wie eine erst kürzlich von Jana Osterkamp verfasste Dissertation gezeigt hat, nicht am Entstehen der tschechoslowakischen Verfassungsgerichtsbarkeit beteiligt; „Vater“ dieser Bestimmungen war vielmehr Jirü Hoetzel, ein entschiedener Gegner Kelsens. ${ }^{11}$

Die Gründe, weshalb die Republik Österreich und die tschechoslowakische Republik fast gleichzeitig eine Verfassungsgerichtsbarkeit einführten, die sich so deutlich vom amerikanischen Modell abhob, müssen tiefer liegen, und ihre Wurzeln sind im Staatsrecht der Habsburgermonarchie zu suchen, aus der beide Republiken hervorgegangen sind. ${ }^{12}$

Dabei ist von vornherein nur die österreichische Hälfte der österreichisch-ungarischen Monarchie in Betracht zu ziehen. ${ }^{13}$ Das Königreich Ungarn besaß - so wie Großbritannien überhaupt keine Verfassung im formellen Sinn, und damit mangelte es schon an der Grundvoraussetzung einer Verfassungsgerichtsbarkeit. Tatsächlich wurde in Ungarn erst 1989 ein Verfassungsgerichtshof gegründet, und Großbritannien besitzt als eines von wenigen europäischen Ländern bis heute weder eine Verfassung noch eine Verfassungsgerichtsbarkeit. ${ }^{14}$ Im Kaisertum Österreich dagegen waren 1867 fünf Staatsgrundgesetze erlassen worden, die in ihrer Gesamtheit die Verfassung ausmachten; Änderungen dieser Verfassung konnten nur vorgenommen werden,

\footnotetext{
${ }^{9}$ HAASE - StRUger, Verfassungsgerichtsbarkeit in Europa 37; SCHUlZ, Verfassungsgerichtsbarkeit im globalen Kontext 2 f; STOURZH, Der Umfang der österreichischen Geschichte 171.

${ }^{10}$ SPÁČIL, Ústavní Soud České Repbuliky 16; SCHELLE et al, Grundriss der Tschechoslowakischen Rechtsgeschichte 22; HELLER, Der Verfassungsgerichtshof 186.

11 OsterkAMP, Verfassungsgerichtsbarkeit in der Tschechoslowakei 10. Sie widerlegt damit eine weitverbreitete Ansicht, wie sie etwa noch von HAASE - STRUGER, Verfassungsgerichtsbarkeit in Europa 58 geteilt wird.

12 OSTERKAMP, Verfassungshüter ohne politischen Rückhalt 276.

13 Siehe für einen allgemeinen Überblick über die Verfassungssituation RUMPLER, Die Habsburgermonarchie, mit weiteren Nachweisen.

${ }^{14}$ HAASE - STRUGER, Verfassungsgerichtsbarkeit in Europa 134, 267. Allerdings ist nicht einmal das UK immun gegen eine Verfassungsgerichtsbarkeit. Beachte die aktuellen Tendenzen im UK, die eine Auswirkung des EU-Rechts, vor allem aber des Human Rights Act 1998 sind; siehe dazu WoOdHOusE, The Constitutional and Political Implications 152-153; ELLIOTT, Embracing “Constitutional” Legislation 35.
} 
wenn in beiden Häusern des Reichsrates wenigstens zwei Drittel der anwesenden Mitglieder dafür stimmten. Solcherart beschlossene Gesetze galten ebenso als Bestandteil der Verfassung; das gesamte Verfassungsrecht war daher schon am Ende der Monarchie über eine Unzahl von Gesetzen verstreut, die immer mehr Details festschrieben - ein Zustand, der in der Republik Österreich nicht nur beibehalten, sondern noch verstärkt wurde, sodass das österreichische Verfassungsrecht bis heute von einer weltweit einzigartigen Zersplitterung gekennzeichnet ist. ${ }^{15}$

Nun könnte man meinen, dass die Unterscheidung von einfachen Gesetzen, die mit einfacher Mehrheit beschlossen wurden, und von Verfassungsgesetzen, die mit qualifizierter Mehrheit beschlossen wurden, zur Folge hätte, dass Gesetze, die den Verfassungsgesetzen widersprechen, void seien. Dies aber war nicht der Fall. Vielmehr war es den Gerichten ausdrücklich verboten, Gesetze zu überprüfen; ${ }^{16}$ die amerikanische „Unsitte“ der judicial review war in Österreich also wohl bekannt, wurde aber abgelehnt. ${ }^{17}$ Damit jedoch war die Unterscheidung zwischen Verfassungsgesetzen und einfachen Gesetzen juristisch ohne Bedeutung; ${ }^{18}$ dies kritisierte der Jurist Georg Jellinek schon 1885 und forderte einen „Verfassungsgerichtshof für Österreich“.19 Er brachte mehrere Beispiele, in denen Abgeordnete darauf hingewiesen hatten, dass ein zu beschließendes Gesetz gegen die Verfassung verstoße, dass es daher einer Zweidrittelmehrheit bedürfe, um dieses Gesetz in Verfassungsrang zu heben. Die Entscheidung aber, ob ein Gesetz einer einfachen Mehrheit oder einer Zweidrittelmehrheit bedürfe, kam allein dem Parlamentspräsidenten zu - und dieser wurde mit einfacher Mehrheit gewählt! Somit hatte es faktisch die einfache Mehrheit der Abgeordneten in der Hand, Gesetze zu beschließen, die von der Verfassung abwichen, aber dennoch - mangels judicial review - unanfechtbar waren.

Georg Jellinek war der Ansicht, dass das Parlament, wenn es mit einfacher Mehrheit Gesetze beschließe, für die eine qualifizierte Mehrheit nötig seien, seine Kompetenzen überschreite, und forderte daher eine gerichtliche Entscheidung über einen derartigen Kompetenzstreit. ${ }^{20}$ Diese Argumentation ist insoweit verständlich, als es zu jener Zeit bereits einen Gerichtshof in Österreich gab, der über Kompetenzstreitigkeiten entschied: Das 1869 errichtete Reichsgericht. ${ }^{21}$ Dieses war schon bei anderer Gelegenheit als Verfassungsgerichtshof bezeichnet worden, ${ }^{22}$ denn obwohl es

\footnotetext{
${ }^{15}$ HeLLER, Der Verfassungsgerichtshof 95; siehe auch STOURZH, Der Umfang der österreichischen Geschichte 170.

16 Artikel 7 Staatsgrundgesetz über die richterliche Gewalt vom 21.12.1867, Reichsgesetzblatt für das Kaiserthum Oesterreich Nr 144/1867.

${ }^{17}$ Dies wurde insbesondere thematisiert, als der Kaiser 1913, im Zusammenhang mit dem Nationalitätenkonflikt in Böhmen, ein „Patent” erließ, also eine Norm, die bewusst weder Gesetz noch Verordnung war, sodass das Reichsgericht und der Verwaltungsgerichtshof vor die Frage gestellt wurden, ob sie dieses Patent überprüfen können, letztlich aber verneinten. Diese weithin unbekannte, aber wichtige Episode in der Geschichte der Verfassungsgerichtsbarkeit wird thematisiert von STOURZH, Der Umfang der österreichischen Geschichte 139. Hinzuzufügen ist, dass auch der junge Hans Kelsen einen anonymen Zeitungsartikel zu dieser Thematik veröffentlichte: Die böhmische Verwaltungskommission vor dem Verwaltungsgerichtshof, Neue Freie Presse Nr. 17.673 vom 5. 11. $1913,3$.

${ }^{18}$ KELSEN, Reichsgesetz und Landesgesetz nach der österreichischen Verfassung 202, 390.

19 JELLINEK, Ein Verfassungsgerichtshof für Österreich. Siehe OLECHOWSKI, Grundrechte und ihr Schutz in der Habsburgermonarchie 35; OSTERKAMP, Verfassungshüter ohne politischen Rückhalt 280.

${ }^{20}$ JELLINEK, Ein Verfassungsgerichtshof für Österreich 20; HELLER, Der Verfassungsgerichtshof 131; OLECHOWSKI, Grundrechte und ihr Schutz in der Habsburgermonarchie 36.

${ }^{21}$ HeLLER, Der Verfassungsgerichtshof 99; OLECHOWSKI, Grundrechte und ihr Schutz in der Habsburgermonarchie 32.

${ }^{22}$ HeLLER, Der Verfassungsgerichtshof 127; JABLONER, Die Gerichtshöfe des öffentlichen Rechts 220.
} 
kein Recht auf eine judicial review besaß, hatte es doch eine Reihe anderer Funktionen, die auf den Schutz der Verfassung ausgerichtet waren. Außer dem Recht, über Kompetenzkonflikte zu entscheiden, ist hier vor allem das Recht, Verwaltungsakte auf ihre Verfassungsmäßigkeit zu prüfen, zu nennen. Gerade hier aber zeigte sich die ganze Problematik einer fehlenden Verfassungsgerichtsbarkeit: So hatte etwa im Jahr 1883 ein Zeitungsherausgeber Beschwerde beim Reichsgericht erhoben, weil die Behörde ihm das Recht, seine Zeitung in Trafiken (Zeitungshandlungen) $\mathrm{zu}$ verkaufen, entzogen hatte, und er berief sich dabei auf die in der Verfassung gewährleistete Pressefreiheit. Der Reichsgericht musste jedoch feststellen, dass die Behörde ihre Handlung auf eine entsprechende Bestimmung des Pressegesetzes 1862 stützen konnte; eine Prüfung, ob das Pressegesetz 1862 selbst mit der Verfassung im Widerspruch stand, fand nicht statt. ${ }^{23}$

Als Hans Kelsen wenige Wochen nach Ausrufung der Republik, im Herbst 1918, vom neuen Staatskanzler Karl Renner beauftragt wurde, ein Gesetz vorzubereiten, mit dem die Kompetenzen des ehemaligen Reichsgericht auf ein neues, republikanisches Gericht übertragen werden sollten, schlug er als Namen für dieses Gericht Verfassungsgericht vor. ${ }^{24}$ Und er stellte fest, dass es ein großes Bedürfnis „nach einem Gerichte" gebe, ,das nach jeder Richtung dem Schutze der Verfassung dient". 25 Vorläufig wurden dem Verfassungsgerichtshof nur die Kompetenzen des ehemaligen Reichsgerichts übertragen, ${ }^{26}$ doch bei den weiteren Reformen bis hin zur Bundesverfassung von 1920 wurden diese Kompetenzen beständig vermehrt. Dies hing mit dem Hauptproblem der Verfassungsarbeiten in Österreich, der Umwandlung in einen Bundesstaat, zusammen. ${ }^{27}$ Noch während des Ersten Weltkrieges hatte Renner die Idee geäußert, Österreich in einen Bundesstaat umzuwandeln. Bund und Länder sollten jeweils eigenständige Parlamente erhalten; die Frage der Kompetenzabgrenzung wurde damit immer wichtiger. Renner propagierte ein „Verfassungsgericht des Bundes", welches auf Antrag der Bundesregierung feststellen könne, ob ein Gesetzgebungsakt in die Kompetenz des Bundes oder der Länder fallen solle. ${ }^{28}$

Zwischen der Ausrufung der Republik 1918 und der Beschlussfassung über das BundesVerfassungsgesetz 1920 bestand ein zweijähriges Verfassungsprovisorium. Österreich war zwar kein echter Bundesstaat, gliederte sich aber bereits in Länder, die eigene Landesversammlungen mit einem Gesetzgebungsrecht besaßen. ${ }^{29}$ Im Auftrag Renners erstellte Kelsen im Jänner oder Februar 1919 ein Gutachten, in dem er den Plan, Österreich in einen Bundesstaat umzuwandeln, sowie mögliche Alternativen erörterte. In diesem Zusammenhang schlug er vor, einen

\footnotetext{
${ }^{23}$ OleCHOwSKI, Grundrechte und ihr Schutz in der Habsburgermonarchie 34.

${ }^{24}$ Der exakte Name für das neue Gericht, den Kelsen vorschlug, lautete in deutscher Sprache Verfassungsgericht; Staatskanzler KARL RENNER änderte dies in Verfassungsgerichtshof, was in der Sache aber keinen Unterschied macht. ${ }^{25}$ STOURZH, Wege zur Grundrechtsdemokratie 314; SCHMITZ, The Constitutional Court of the Republic of Austria 244; OLECHOWSKI, Der Beitrag Hans Kelsens zur österreichischen Bundesverfassung 214.

26 Gesetz vom 25. 1. 1919 über die Errichtung eines deutschösterreichischen Verfassungsgerichtshofes, Staatsgesetzblatt für die Republik Deutschösterreich Nr. 48/1919; siehe HELLER, Der Verfassungsgerichtshof 149.

27 ÖHLINGER, The Genesis of the Austrian Model 212. Der Autor hält die Diskussion über Föderalismus für essentiell für die Anfänge der Verfassungsgerichtsbarkeit in Österreich und sagt, dass die „power oft he Constitutional Court was obviously designed as an instrument to resolve conflicts between federal and state law".

${ }^{28}$ JABLONER, Die Gerichtshöfe des öffentlichen Rechts 221; ÖHLINGER, The Genesis of the Austrian Model 211; SCHMITZ, The Constitutional Court of the Republic of Austria 242.

${ }^{29}$ Siehe für Details MERKL, Die Verfassung der Republik Deutschösterreich.
} 
Staatsverfassungsgerichtshof zu schaffen, der Landesgesetze aufheben sollte, wenn sie gegen Gesetze des Gesamtstaates verstießen. ${ }^{30}$ Noch kurz bevor das Gutachten in der „Zeitschrift für Öffentliches Recht" veröffentlicht wurde, ${ }^{31}$ griff die Konstituierende Nationalversammlung diesen Vorschlag auf und änderte die provisorische Verfassung dahin ab, dass Gesetzesbeschlüsse der Landesversammlungen noch vor ihrer Promulgation der Staatsregierung bekanntgegeben werden müssten und diese das Recht habe, diese innerhalb von 14 Tagen beim Verfassungsgerichtshof wegen Verfassungswidrigkeit zu bekämpfen. ${ }^{32}$ Tatsächlich kam es in den etwa eineinhalb Jahren, in denen diese Regelung in Geltung stand, immerhin zweimal zu solchen Fällen. ${ }^{33}$ Sie war freilich insofern problematisch, als sie zwar dem Bund die Möglichkeit gab, Landesgesetze zu bekämpfen, nicht aber den Ländern dieses Recht in Bezug auf Bundesgesetze. Auf dieses Problem wies der Tiroler Jurist Stephan Falser hin; im Oktober 1919 erstellte er selbst einen stark föderalistischen Verfassungsentwurf, in dem er ein wechselseitiges Anfechtungsrecht für Bund und Länder vorsah. ${ }^{34}$

Diese Regelung ging letztlich auch in den endgültigen Text des BundesVerfassungsgesetzes 1920 ein (Artikel 140). Die bis heute aufrechte Regelung ist von hoher theoretischer Bedeutung für die bundesstaatliche Struktur Österreichs, in der Praxis wurde sie bislang nur selten angewendet. Der eigentliche Grund für den späteren Siegeszug des österreichischen Modells der Verfassungsgerichtsbarkeit ist woanders zu suchen: Der Verfassungsgerichtshof wurde in Artikel 140 Bundes-Verfassungsgesetz nämlich auch dazu ermächtigt, selbst ein Gesetzesprüfungsverfahren einzuleiten, wenn er - in irgendeinem anderen Verfahren - ein Gesetz anzuwenden hatte, an dessen Verfassungsmäßigkeit Zweifel bestünden.

Diese Textierung stammt von Hans Kelsen. Kelsens entscheidender Einfluss auf das BundesVerfassungsgesetz resultiert nicht nur aus dem Umstand, dass er im Sommer 1919 jene Entwürfe schrieb, auf denen die weiteren Verfassungsarbeiten aufbauten, ${ }^{35}$ er wurde auch im Juli 1920 - als parteiunabhängiger Verfassungsexperte - dem parlamentarischen Unterausschuss beigezogen, der bis Oktober den endgültigen Verfassungstext beschloss. In diesem Ausschuss brachte Kelsen am 31. August den Vorschlag, dass der Verfassungsgerichtshof ein Gesetzesprüfungsverfahren „von Amts wegen", aus eigener Initiative, starten konnte. ${ }^{36}$ Diese Formulierung war den Abgeordneten jedoch zu weitgehend. Der Verfassungsgerichtshof hätte nach Belieben sämtliche Gesetze jederzeit einer Prüfung unterziehen können. Daher regte Kelsen am 23. September eine neue Formulierung an: „Der Verfassungsgerichtshof erkennt über Gesetzwidrigkeit von Verordnungen einer Bundes- oder einer

\footnotetext{
30 OleChOwsKI, Der Beitrag Hans Kelsens zur österreichischen Bundesverfassung 215-216; JABLONER, Die Gerichtshöfe des öffentlichen Rechts 224.

${ }^{31}$ KELSEN, Die Stellung der Länder in der künftigen Verfassung Deutschösterreichs.

${ }^{32}$ Gesetz vom 14. 3. 1919 über die Volksvertretung, Staatsgesetzblatt Nr. 179/1919, Artikel 13 und 15. Siehe SCHMITZ, The Constitutional Court of the Republic of Austria 247.

${ }^{33}$ Die Quellenlage hierzu ist sehr dürftig; vgl näher JABLONER, Die Gerichtshöfe des öffentlichen Rechts 224.

34 STOURZH, Wege zur Grundrechtsdemokratie 332; OlECHOWSKI, Der Beitrag Hans Kelsens zur österreichischen Bundesverfassung 227.

35 STOURZH, Wege zur Grundrechtsdemokratie 317; OLECHOwSKI, Der Beitrag Hans Kelsens zur österreichischen Bundesverfassung 216.

36 ÖHLIngER, The Genesis of the Austrian Model 213; OlECHOwsKI, Der Beitrag Hans Kelsens zur österreichischen Bundesverfassung 227.
} 
Landesbebörde auf Antrag eines Gerichtes, soferne aber eine solche Verordnung die Voraussetzung eines Erkenntnisses des Verfassungsgerichtshofes bilden soll, von Amts wegen". ${ }^{37}$

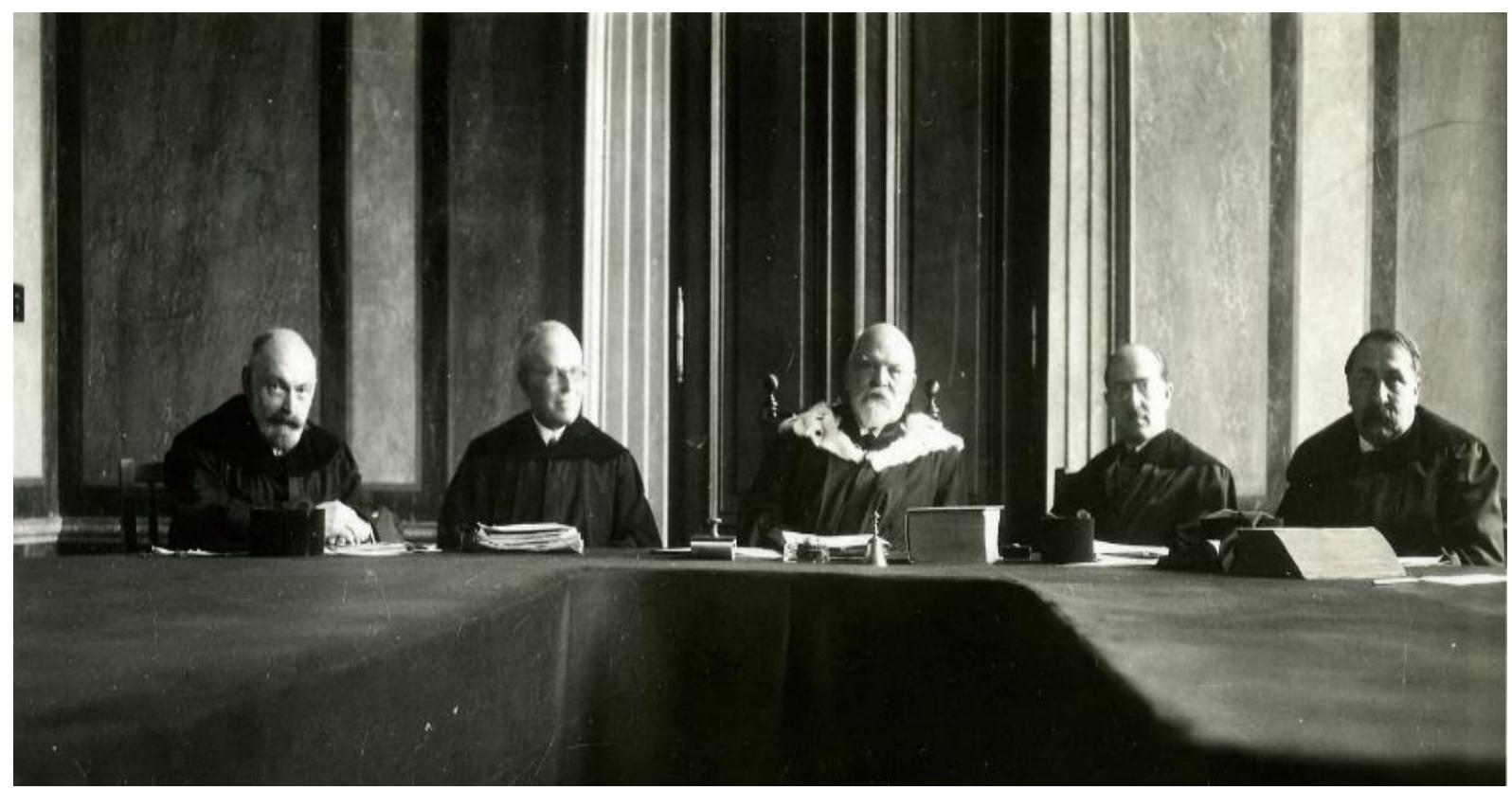

1. Abbildung: Sitzung des österreichischen Verfassungsgerichtshofes, um 1925. In der Mitte der Präsident, Paul Vittorelli, zweiter von rechts: Hans Kelsen. Mit freundlicher Genehmigung von Anne Feder Lee, Honolulu.

Es war buchstäblich in letzter Minute: Eine Woche später, am 1. Oktober 1920, fand die förmliche Beschlussfassung der Konstitutierenden Nationalversammlung über das BundesVerfassungsgesetz statt, und die genannte Bestimmung wurde in der oben zitierten Fassung - als Artikel 140 Absatz 1 - angenommen. ${ }^{38}$

Was dies für die Praxis bedeutete, sei verdeutlicht, wenn wir den oben beschriebenen Fall noch einmal Revue passieren lassen: Wenn sich nunmehr ein Zeitungsherausgeber auf die Pressefreiheit berief und eine Behörde behauptete, dass ihre Maßnahme durch das Pressegesetz 1862 gedeckt sei, so hatte der Verfassungsgerichtshof zunächst ein Verfahren zur Prüfung der verwaltungsbehördlichen Maßnahme einzuleiten. Wenn den Richtern während dieses Verfahrens Zweifel an der Verfassungsmäßigkeit des Pressegesetzes kamen, so hatten sie das erste Verfahren zu unterbrechen und von Amts wegen (ex officio) ein neues Verfahren zur Prüfung des Pressegesetzes selbst einzuleiten und gegebenenfalls die Verfassungswidrigkeit des Gesetzes festzustellen. Erst nach Ausgang dieses zweiten Verfahrens konnte das erste Verfahren fortgesetzt werden - und falls in der Zwischenzeit das Gesetz aufgehoben worden war, so wurde nun in aller Regel auch die behördliche Maßnahme aufgehoben.

Diese Fallkonstellation erinnert an das amerikanische System, doch ist festzuhalten, dass das österreichische System einen ganz anderen Entstehungshintergrund hatte und aus einem Streit

\footnotetext{
${ }^{37}$ ERMACORA, Quellen zum österreichischen Verfassungsrecht 495.

38 Gesetz womit die Republik Österreich als Bundesstaat eingerichtet wird (Bundes-Verfassungsgesetz) vom 1. 10. 1920, Artikel 140, Bundesgesetzblatt für die Republik Österreich Nr. 1/1920; siehe ÖHLINGER, The Genesis of the Austrian Model 213; OLECHOWSKI, Der Beitrag Hans Kelsens zur österreichischen Bundesverfassung 227.
} 
um die Föderalisierung Österreichs hervorgegangen war. Dies ist auch der Hauptgrund, dass der Verfassungsgerichtshof das einzige Gericht in Österreich war, das über die Verfassungswidrigkeit von Gesetzen entscheiden konnte, und anderen Gerichten eine judicial review ausdrücklich verboten war. Befand der Verfassungsgerichtshof, dass das Gesetz verfassungswidrig war, so hob er es auf, was mit allgemeiner Wirkung im Bundesgesetzblatt kundgemacht wurde. Bis zu seiner Entscheidung aber blieb das Gesetz in Kraft - obwohl es verfassungswidrig war, war es also nicht void, sondern bloß anfechtbar, nicht absolut, sondern nur relativ nichtig. ${ }^{39}$

Die Entwicklung in der Tschechoslowakei wich von der Entwicklung in Österreich in vielen Punkten ab: ${ }^{40}$ Obwohl auch sie aus sehr verschiedenen Teilen der Habsburgermonarchie zusammengesetzt war, war die Tschechoslowakei kein Bundesstaat, sondern ein Einheitsstaat; ein Bedürfnis, Landes- oder Bundesgesetze auf ihre Übereinstimmung mit einer Bundesverfassung zu überprüfen, bestand nicht. Ferner war die Gerichtsorganisation der Habsburgermonarchie in der Tschechoslowakei genauso wie in der Republik Österreich weitgehend übernommen worden, doch war der tschechoslowakische Gesetzgeber 1918 den Weg gegangen, für die Kompetenzen des ehemaligen Reichsgerichts kein eigenes Gericht $\mathrm{zu}$ schaffen, sondern dem Obersten Verwaltungsgericht (Nejyy šs ś správni soud) ${ }^{41}$ zu übertragen. Als man dann 1920 zur Errichtung des Verfassungsgerichts schritt, ${ }^{42}$ erhielt dieses lediglich die Kompetenz, über die Verfassungsmäßigkeit von Gesetzen zu entscheiden, während dies in Österreich nur eine, wenn auch zentrale, Kompetenz des Verfassungsgerichtshofes ist. ${ }^{43}$ Woran es also mangelte, war das „Material“, waren die Anlassfälle, in denen das tschechoslowakische Gericht entscheiden konnte. Nur wenige Behörden waren befugt, einen Prüfungsantrag zu stellen, und diese hatten in der Praxis aus verschiedenen Gründen nur wenig Interesse an einer Aktivierung der tschechoslowakischen Verfassungsgerichtsbarkeit. Und tatsächlich entfaltete dieses Gericht eine äußerst geringe Aktivität, sodass in der Literatur bis vor kurzem sogar unklar war, ob das Gericht jemals aktiv geworden sei. Die bereits erwähnte Studie von Osterkamp hat hier Klarheit geschaffen: Tatsächlich kam es ein einziges Mal zu einer Gesetzesaufhebung, und dies erfolgte nach einem jahrelangen Verfahren grotesker Weise erst im Jahre 1939, als die Tschechoslowakei bereits nicht mehr existierte. ${ }^{44}$

\section{3. „Der Hüter der Verfassung”}

In der europäischen Staatslehre erschien daher der österreichische Verfassungsgerichtshof als einziger seiner Art und erregte großes Aufsehen. Als die Vereinigung der Deutschen Staatsrechtslehrer im Jahr 1928 in Wien tagte, wurde die Frage der Staatsgerichtsbarkeit von zwei Referenten behandelt, neben dem Gastgeber Kelsen war es der Berliner Verfassungsrechtler Heinrich Triepel, der vom Vorstand gebeten wurde, ein Referat zu halten. Doch kam es nicht zur beabsichtigten großen Kontroverse; Triepel hatte seinen Gegenstand ganz anders aufgefasst, sprach

\footnotetext{
${ }^{39}$ KELSEN, Mitbericht 71 (41).

${ }^{40}$ Ein guter Vergleich ist zu finden bei OSTERKAMP, Verfassungshüter ohne politischen Rückhalt $281 \mathrm{f}$.

${ }^{41}$ Zákon o nejvyšším správním soudě, 2.11. 1918, Sbírce zákonů a nařízení, Nr 3/1918.

42 Zákon kterým se uvozuje Ústavní listina Československé republiky, 29. 2. 1920, Sbírce zákonů a nařízení, Nr. $121 / 1920$.

${ }^{43}$ HAASE - STRUGER, Verfassungsgerichtsbarkeit in Europa 60.

${ }^{44}$ OSTERKAMP, Verfassungsgerichtsbarkeit in der Tschechoslowakei 184.
} 
ausführlich über Ministeranklagen und widmete sich der Frage von verfassungswidrigen Gesetzen nur am Rande. Gemeinsam mit Kelsen bekannte er ein, dass sie von zwei Seiten „Schneisen in einen Wald" getrieben hätten, ohne einander zu begegnen. ${ }^{45}$

So folgte die große Kontroverse erst ein knappes Jahr später, als der Berliner Professor Carl Schmitt, der bei der Wiener Tagung gefehlt hatte, einen Aufsatz über den „Hüter der Verfassung“ veröffentlichte. ${ }^{46}$ In dieser Arbeit sprach er der Justiz die Möglichkeit, über Verfassungsfragen zu entscheiden, schlichtweg ab. Die Aufgabe der Justiz sei es, in konkreten Einzelfällen Recht zu sprechen, Gesetze auf einen Sachverhalt anzuwenden. Bei der Entscheidung über Verfassungsstreitigkeiten gebe es aber keinen Sachverhalt, der unter ein Gesetz subsumiert werden könne. ${ }^{47}$ Zudem aber ortete Schmitt - so wie schon davor Triepel - einen scharfen Gegensatz zwischen Rechtsfragen und politischen Fragen; ein Gericht aber, das politische Fragen entscheide, sei kein Gericht mehr.

Kelsen reagierte 1931 auf diese Arbeit mit seiner Gegenschrift ,Wer soll der Hüter der Verfassung sein?". ${ }^{48}$ Gemeinsam mit der Veröffentlichung seiner 1928 gehaltenen Rede wurde dieser Aufsatz zu einer allgemein-theoretischen Rechtfertigung der österreichischen Verfassungsgerichtsbarkeit; er hob die für Österreich geschaffene Rechtslage auf ein abstraktes Niveau und machte sie so zu einem Modell, das für viele andere Rechtsordnungen das Vorbild abgab. ${ }^{49}$

Das Bild, das Kelsen dabei verwendete, erinnert durchaus bis zu einem gewissen Grad an jenes des eingangs zitierten US-Supreme Court, der von der Verfassung als einem superior, paramount law gesprochen hatte. Auch Kelsen sah in der Verfassung eine oberste Norm; ${ }^{50}$ er erweiterte dieses Bild aber noch, indem er die gesamte Rechtsordnung hierarchisch gliederte und erklärte, dass die Verfassung in derselben Weise dem Gesetz übergeordnet sei wie das Gesetz einem Gerichtsurteil. Sowohl wenn das Parlament ein neues Gesetz erlasse, als auch, wenn ein Richter ein Urteil spreche, müssen immer zuerst die höherrangigen Normen untersucht werden, die zur Erzeugung eines neuen Gesetzes, eines neuen Gerichtsurteiles ermächtigen. Diese höherrangigen Normen geben gewissermaßen einen Rahmen vor, der aber zumeist offen ist, dem rechtssetzenden Organ einen Spielraum lasse. Wie sich das Organ dann konkret entscheide, obliege seinem Ermessen, hier ist der Boden juristischer Interpretation verlassen und der von Zweckmäßigkeitserwägungen - damit aber: von politischen Erwägungen - erreicht. Beides, rechtliche Interpretation und politische Abwägung, sei immer vorhanden, wenn ein Rechtsakt geschaffen werde; Gesetzgebung und Urteilsschöpfung seien daher vom Wesen her dasselbe. ${ }^{51}$

Der politische Hintergrund der Kelsen-Schmitt-Kontrovese wird besonders deutlich, wenn man liest, wen Schmitt als das zum Hüter der Verfassung berufene Organ ansah: Es sollte dies der Reichspräsident sein, gewählt vom gesamten Volk und Inhaber einer , neutralen Macbt“ ${ }^{52}$ Kelsen hielt

45 Triepel, Bericht 9; Kelsen, Mitbericht; Olechowski, Hans Kelsen als Mitglied der Deutschen Staatsrechtslehrervereinigung.

${ }^{46}$ Der Artikel, der zuerst im Archiv des öffentlichen Rechts 1929 publiziert wurde, wurde später zu einer Monographie erweitert: SCHMITT, Der Hüter der Verfassung. Siehe zu der Kontroverse im Allgemeinen DinER/STOLLEIS (1999).

47 SCHMiTT, Der Hüter der Verfassung 36 ff.

${ }^{48}$ KELSEN, Wer soll der Hüter der Verfassung sein?

${ }^{49}$ VAN OOYEN, Die Funktion der Verfassungsgerichtsbarkeit VIII.

${ }^{50}$ KeLsEN, Mitbericht 36 (7).

${ }^{51}$ KELSEN, Wer soll der Hüter der Verfassung sein 586 (67).

52 SCHMIT'T, Der Hüter der Verfassung 132. 
den Reichspräsidenten für gänzlich ungeeignet, zumal doch er selbst eines derjenigen Organe sei, dessen Akte auf ihre Verfassungsmäßigkeit zu kontrollieren sei. ${ }^{53}$

Zum Zeitpunkt der Debatte, 1931, war das parlamentarische System der Weimarer Republik schon so gut wie gescheitert, die Regierungen stützten sich weitgehend auf das Notverordnungsrecht des Reichspräsidenten. Eine Ähnliche Entwicklung erfolgte in vielen anderen Staaten. Im März 1933 führte die österreichische Bundesregierung einen Staatsstreich durch, zum Teil, weil sie hoffte, auf diese Weise einen Sieg der Nationalsozialisten bei den nächsten Parlamentswahlen zu verhindern, zum Teil aber auch, weil sie selbst das parlamentarischdemokratische System ablehnte. Auch sie begann, mit Notverordnungen zu regieren; als der Verfassungsgerichtshof angerufen wurde, die Gültigkeit dieser Notverordnungen zu überprüfen, wurde auch er ausgeschaltet. ${ }^{54}$ Vielleicht zeigt dies besser als jede theoretische Diskussion den engen Zusammenhang zwischen Demokratie und Verfassungsgerichtsbarkeit.

\section{Die Entwicklung nach 1945}

Nach dem Ende des 2. Weltkrieges began der Triumphzug der Verfassungsgerichtsbarkeit in Europa. ${ }^{55}$ Schon 1945 wurde der österreichische Verfassungsgerichtshof wiedererrichtet, ein Jahr später folgte, nach dem österreichischen Vorbild, der bayrische Verfassungsgerichtshof, der selbst wieder das Vorbild für das deutsche Bundesverfassungsgericht war, das 1951 seine Tätigkeit aufnahm. Auch die italienische Verfassung von 1948 sah einen Verfassungsgerichtshof vor, der 1956 seine Tätigkeit aufnahm. Acht Jahre später, 1964, folgte Jugoslawien als erster sozialistischer Staat, der eine Verfassungsgerichtsbarkeit einführte. In der Tschechoslowakei erfolgte die Rückkehr zur Verfassungsgerichtsbarkeit nicht vor $1992,{ }^{56}$ die gemeinsame Tradition wird nun unabhängig voneinander in Prag und in Bratislava fortgeführt. Und mittlerweile gibt es kaum einen europäischen Staat ohne Verfassungsgerichtshof, einen obersten Gerichtshof mit verfassungsgerichtlichen Kompetenzen oder eine andere Variante der Verfassungsgerichtsbarkeit, die moisten von ihnen aber folgen dem Kelsenianischen Modell.

Und Europa selbst? Bekanntlich wurde das Projekt eines Europäischen Verfassungsvertrages 2004 nicht realisiert. Nichtsdestoweniger können die Gründungsverträge der Europäischen Union (die Verträge von Rom, von Maastricht, von Lissabon usw.) durchaus als seine Verfassung der EU und der Europäische Gerichtshof in Luxemburg al sein Verfassungsgerichtshof angesehen werden. ${ }^{57}$ Insbesondere die Nichtigerklärung von Rechtsakten nach Artikel 264 des Vertrages über die Arbeitsweise der EU zeigt viele Parallelen zur Nichtigkerklärung von Gesetzen nach Artikel 140 der österreichischen BundesVerfassungsgesetzes. In der europarechtlichen Literatur wird die Europäische Kommission

\footnotetext{
${ }^{53}$ KELSEN, Wer soll der Hüter der Verfassung sein 577 (58).

${ }^{54}$ WALTER, Die Ausschaltung des Verfassungsgerichtshofes.

55 Heller, Der Verfassungsgerichtshof 74. Der hier gegebene Überblick folgt HAASE - STRUGER, Verfassungsgerichtsbarkeit in Europa 69 ff. Siehe für Deutschland auch BOROWSKI, The Beginnings of Germany's Federal Constitutional Court, mit weiteren Nachweisen.

${ }^{56}$ Der Verfassungsgerichtshof der Tschechoslowakischen Republik bestand nur zehn Monate: SpÁčIL, Ústavní Soud České Repbuliky 26.

${ }^{57}$ Dazu schon WeIDMAnN, Der Europäische Gerichtshof.
} 
vielfach als „Hüter der Verträge” beschrieben. ${ }^{58}$ Dieses Bild ist ebenso irreführend wie jenes, dass der deutsche Reichspräsident Hüter der Verfassung sein könne. Bleibt man beim Bild Kelsens, so kommt die Rolle eines "Hüters der Verträge” viel eher dem Europäischen Gerichtshof zu.

\section{Literatur- und Quellenverzeichnis}

BOROwSKI, Martin: The Beginnings of Germany's Federal Constitutional Court. Ratio Juris 16/2003, $155-186$. Diner, Dan and Michael Stolleis (eds) (1999). Hans Kelsen and Carl Schmitt: a Juxtaposition. Gerlingen: Bleicher. ELLIOTT, Mark: Embracing “Constitutional” Legislation: Towards Fundamental Law?. Northern Ireland Legal Quarterly 54(1)/2003 25-42.

ERMACORA, Felix: Quellen zum österreichischen Verfassungsrecht. Wien 1967

HAASE, Gudrun - STRUGER, Katrin: Verfassungsgerichtsbarkeit in Europa. Wien 2009

HELLER, Kurt: Der Verfassungsgerichtshof. Die Entwicklung der Verfassungs- gerichtsbarkeit in Österreich von den Anfängen bis zur Gegenwart. Wien 2010

IPSEN, Hans Peter: Europäisches Gemeinschaftsrecht. Tübingen 1972

JABLONER, Clemens: Die Gerichtshöfe des öffentlichen Rechts im Zuge des Staatsumbaues 1918 bis 1920. Beiträge zur Rechtsgeschichte Österreichs 1/2011, 213-227

JELLINEK, Georg: Ein Verfassungsgerichtshof für Österreich. Wien 1885

KELSEN, Hans: Reichsgesetz und Landesgesetz nach der österreichischen Verfassung. Archiv des öffentlichen Rechts 32/1914, 202-245, 390-438

KELSEN, Hans: Die Stellung der Länder in der künftigen Verfassung Deutschösterreichs. Zeitschrift für öffentliches Recht 1/1919, 98-122.

KELSEN, Hans: Mitbericht [zum Beratungsgegenstand: Wesen und Entwicklung der Staatsgerichtsbarkeit]. In: Veröffentlichungen der Vereinigung der Deutschen Staatsrechtslehrer 5. Berlin - Leipzig 1929, 30-84. Reprinted in van OOYEN, Robert Christian (ed.): Wer soll der Hüter der Verfassung sein? Tübingen 2008, 157.

KELSEN, Hans: Wer soll der Hüter der Verfassung sein? Die Justiz VI/1931, 576-628. Reprinted in: van OOYEN, Robert Christian (ed.): Wer soll der Hüter der Verfassung sein? Tübingen 2008, 58-105.

MERKL, Adolf: Die Verfassung der Republik Deutschösterreich. Ein kritisch- systematischer Grundriß. Wien - Leipzig 1919

ÖHLInger, Theo: The Genesis of the Austrian Model of Constitutional Review of Legislation. Ratio Juris 16/2003, 206-222

OLECHOWsKI, Thomas: Der Beitrag Hans Kelsens zur österreichischen Bundesverfassung. In: WALTER, Robert et al (eds), Hans Kelsen: Leben - Werk - Wirksamkeit. Wien 2009, 211-230

OleCHOwSKI, Thomas: Grundrechte und ihr Schutz in der Habsburgermonarchie. Österreichische Richterzeitung 88/2010, 30-37

OLECHOWSKI, Thomas: Hans Kelsen als Mitglied der Deutschen Staatsrechtslehrervereinigung. In JESTAEDT, Matthias (ed.): Hans Kelsen und die deutsche Staatsrechtslehre. Tübingen 2013, 11-27.

OSTERKAMP, Jana: Verfassungsgerichtsbarkeit in der Tschechoslowakei (1920-1939). Verfassungsidee Demokratieverständnis - Nationalitätenproblem. Frankfurt am Main 2009

OSTERKAMP, Jana: Verfassungshüter ohne politischen Rückhalt. Das tschechoslowakische Verfassungsgericht nach 1920 im Vergleich mit Österreich. Beiträge zur Rechtsgeschichte Österreichs 1/2011, 275-290

PAUlson, Stanley: Constitutional Review in the United States and Austria: Notes on the Beginnings. Ratio Juris 16/2003, 223-239

RUMPLER, Helmut - URBANITSCH, Peter (eds): Die Habsburgermonarchie 1848-1918 VII: Verfassung und

${ }^{58}$ Diese Phrase geht wohl zurück auf IPSEN, Europäisches Gemeinschaftsrecht 361. 
Parlamentarismus. Wien 2000

SCHELLE, Karel - TAUCHEN, Jaromír: Grundriss der Tschechoslowakischen Rechtsgeschichte. München 2009

SCHePpele, Kim Lane: Constitutional Negotiations. Political Contexts of Judicial Activism in Post-Soviet Europe. International Sociology 18(1)/2003, 219-38.

SCHMITT, Carl: Der Hüter der Verfassung. Tübingen 1931. Reprinted Berlin 1996

SCHMITZ, Georg: The Constitutional Court of the Republic of Austria 1918-1920, Ratio Juris 16/2003, 240-265

SCHULZ, Martin: Verfassungsgerichtsbarkeit im globalen Kontext. giga-focus 5/2010, 1-8

SELLERT, Wolfgang: Richterliches Prüfungsrecht. In: ERLER, Adalbert et al. (eds): Handwörterbuch zur Deutschen Rechtsgeschichte IV. Berlin 1990, 1050-1054.

SPÁČIL, Michal: Ústavní Soud České Repbuliky. The Constitutional Court of the Czech Republic. Brno 2008

STOLLEIS, Michael: Judicial Review, Administrative Review, and Constitutional Review in the Weimar Republic. Ratio Juris 16/2003, 266-280

STOURZH, Gerald: Hans Kelsen, die österreichische Bundesverfassung und die rechtsstaatliche Demokratie. In: Hans Kelsen-Institut (ed.): Die Reine Rechtslehre in wissenschaftlicher Diskussion. Wien 1982, 7-29

STOURZH, Gerald: Wege zur Grundrechtsdemokratie. Wien - Köln - Weimar 1989

STOURZH, Gerald: Der Umfang der österreichischen Geschichte. Wien - Köln - Graz 2011

TRIEPEL, Heinrich: Bericht [zum Beratungsgegenstand: Wesen und Entwicklung der Staatsgerichtsbarkeit]. In: Veröffentlichungen der Vereinigung der Deutschen Staatsrechtslehrer 5. Berlin - Leipzig 1929, 2-28.

Urofsky, Melvin I. - Finkelman, Paul (eds): Documents of American Constitutional and Legal History. Vol. I. 2nd edition. Oxford 2002

van OOYEN, Robert Christian: Die Funktion der Verfassungsgerichtsbarkeit in der pluralistischen Demokratie und die Kontroverse um den ,Hüter der Verfassung'. In: van OOYEN, Robert Christian (ed.). Wer soll der Hüter der Verfassung sein? Tübingen 2008, VII-XXIII

WALTER, Robert: Die Ausschaltung des Verfassungsgerichtshofes im Jahre 1933. In: Verfassungstag 1997 (ed.): Verfassungsgerichtshof der Republik Österreich. Wien 1997, 17-34

WeIDMANN, Klaus W.: Der Europäische Gerichtshof auf dem Weg zu einem europäischen Verfassungsgerichtshof. Frankfurt am Main 1985

Woodhouse, Diana: The Constitutional and Political Implications of a United Kingdom Supreme Court. Legal Studies 24/2004, 134-55 